
December 1941

\title{
THE CARBOHYDRATE CONTENT OF COLLAGEN
}

\author{
By John Beek, Jr.
}

\section{ABSTRACT}

Colorimetric methods are used to study the carbohydrate in preparations of collagen made from hide, tendon, and bone. It was found that the three preparations all contain the same amount of the same kind of carbohydrate. This result confirms the indication of other experiments, namely, that the white fibrous connective tissues of hide, tendon, and bone are chemically identical. The color reactions of the collagen did not correspond exactly to those of any one or mixture of two of the known sugars studied, but they were similar to the reactions of a mixture of glucose and galactose. An attempt to ferment the carbohydrate in the hydrolyzate with galactose-active yeast showed that neither free $d$-glucose nor free $d$-galactose is present. A concentrate of the carbohydrate, prepared by the method described by Rimington, gave color reactions corresponding closely with those of a mixture of glucose and galactose. It appears, then, that the carbohydrate is combined through some difficultly hydrolyzable linkage, or that it is composed of $l$-glucose and $l$-galactose.

\section{CONTENTS}

Pago

1. Introduction

II. Materials and methods of measurement

III. Results_._.

IV. Summary

V. References

\section{INTRODUCTION}

In an investigation now in progress, some properties of the white fibrous connective tissues of the hide, tendons, and bones of a steer are being compared. Measurements of combination with acid and with base [1], ${ }^{1}$ and of electrophoretic mobility [2] gave evidence that in the natural state the white fibrous connective tissues from the three structures are chemically identical. In this paper colorimetric measurements of the carbohydrate content of the three preparations of collagen are described, together with some experiments giving information about the qualitative composition of the carbohydrate.

\section{MATERIALS AND METHODS OF MEASUREMENT}

The procedure used in preparing the collagen was essentially that recommended by Highberger [3]. The slight modifications that were necessary are given in an earlier publication [1]. Solutions for sam-

\footnotetext{
${ }^{1}$ Figures in brackets refer to list of references at end of this paper.
} 
pling were made by hydrolyzing the collagen with dilute sulfuric acid.

The rare sugars used were made available through the courtesy of the Polarimetry Section of the National Bureau of Standards. The gulose was in the form of its addition compound with calcium chloride. All the other sugars used were in the pure crystalline form.

All the measurements were based on the color reactions of sugars with orcinol or $\alpha$-naphthol. The first measurements were made according to the procedure worked out by M. Sørensen and G. Haugaard [4]. The method depenas on the rate of development of color when a solution containing $40 \mathrm{mg}$ of orcinol and not more than $0.2 \mathrm{mg}$ of carbohydrate in $18 \mathrm{ml}$ of 64-percent sulfuric acid is heated at $80^{\circ} \mathrm{C}$. Sørensen and Haugaard recommended that the transmittancies at 430 and $530 \mathrm{~m} \mu$ of solutions heated for six different periods of time up to 30 minutes be measured. The behavior of the ratio of the two corresponding absorbencies as the heating is continued is used to determine the qualitative composition of the carbohydrate. For this series of measurements in the present investigation, the sources of illumination were a mercury arc with filters designed to isolate the lines at 436 and $546 \mathrm{~m} \mu$, and an incandescent lamp with a filter transmitting a band with its center at $501 \mathrm{~m} \mu$. The transmittancy ${ }^{2}$ of cells $1 \mathrm{~cm}$ thick was measured with a Martens photometer. Serious difficulty was encountered in making the measurements for the solutions that were heated for short periods of time, since the results were very sensitive to changes in the conditions of the reaction, and the colors obtained were fugitive. As it was not convenient to make the arrangements necessary for the satisfactory application of this method, it was decided to study the transmittancy over most of the visible spectrum of solutions prepared according to the Sørensen and Haugaard procedure, using 20 minutes for the time of heating. The reference solutions contained quinol in place of orcinol. Quinol was the only phenol among those tried that gave no color reaction with sugars. A Coleman Regional Spectrophotometer was used for the measurements. ${ }^{3}$

The results obtained by these two methods were still insufficient to establish the nature of the carbohydrate in collagen, and thus could not give its amount. Two effects in particular were desired that had not been obtained. One was a sharp distinction between the shapes of the curves obtained with glucose and galactose; the other was an increased absorption in the region of longer wavelengths, where the measurements are more precise and where discoloration of the protein during hydrolysis or during the reaction would be expected to interfere less. It was found that by using sulfuric acid at a higher concentration and permitting the reaction to take place at $25^{\circ} \mathrm{C}$, red solutions were obtained which had transmittancies much higher in the blue and much lower in the yellow. In addition, the difference between glucose and galactose was somewhat more marked than with the solutions that were heated. The most favorable concentration of sulfuric acid was found to be 73 percent. The solutions were

\footnotetext{
The transmittancy is the ratio of the intensity of the light transmitted by two soiutions that are illuminated with light of the same intensity. The two solutions differ only in the absence from the reference solution of the substance being investigated. The absorbency is the negative common logarithm of the transmittancy. For the purpose of the present work, the absorbency was corrected for the effect of the reagents by subtracting the absorbency obtained with blank solutions, containing all components of the

experimental solutions except the sugar or collagen.
The use of this instrument was made possible through the cooperation of the Office of Forest Pathology of the Bureau of Plant Industry, U. S. Department of Agriculture.
} 
allowed to stand 20 hours before the measurements were made, and quinol was used in the reference solutions.

Another color reaction of sugars with orcinol was investigated and modified for some further measurements. About $0.2 \mathrm{mg}$ of sugar was allowed to react with $20 \mathrm{mg}$ of orcinol and $1.4 \mathrm{mg}$ of ferric chloride in about $18 \mathrm{ml}$ of 36-percent hydrochloric acid. The solutions were allowed to stand for 3 days at $25^{\circ} \mathrm{C}$. It was found later that although the absorbencies were less, a shorter time of reaction gave a better distinction between glucose and galactose. The solutions obtained were green, with a maximum absorbency at about $675 \mathrm{~m} \mu$.

The color reactions of several other phenols were tested to determine their applicability to this problem, but $\alpha$-naphthol was the only

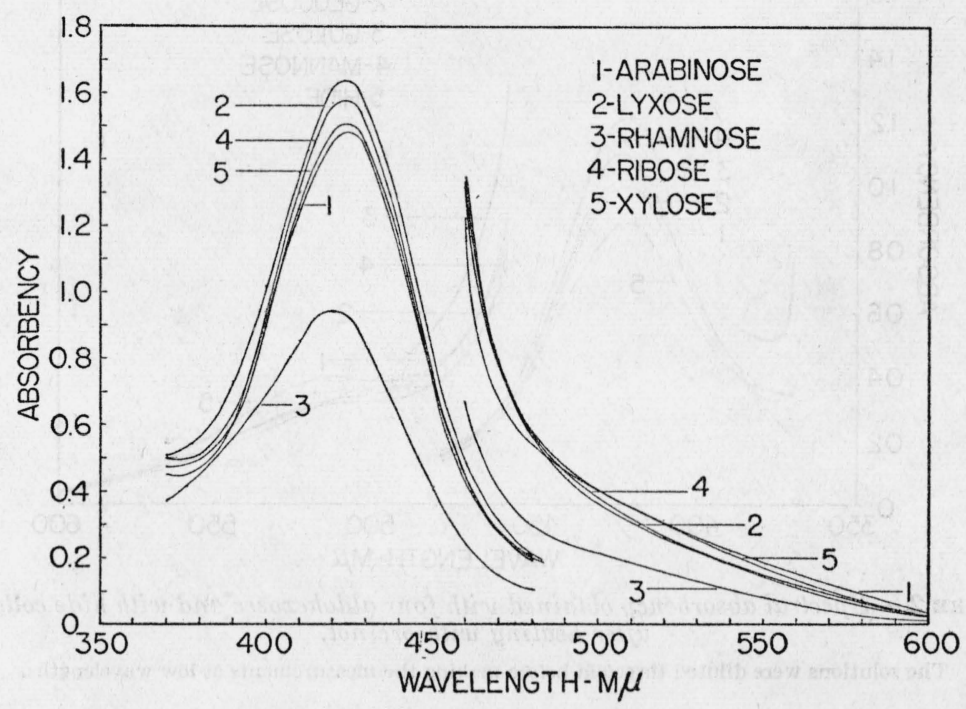

FIGURE 1.-Spectral absorbency obtained with the aldopentoses and with rhamnose, after heating with orcinol.

The solutions were diluted threefold before making the measurements at low wavelengths.

substance that was found to be useful. The conditions for the reaction with $\alpha$-naphthol were like those used with the orcinol solutions that were heated, except that the temperature was $100^{\circ} \mathrm{C}$. The transmittancies were measured with reference to water.

\section{RESULTS}

The spectral absorbency of a series of sugars and of hide collagen heated with orcinol ${ }^{4}$ is shown in figures 1 and 2 . The collagen solution contained the hydrolyzate from $34 \mathrm{mg}$ of dry collagen. The measurements in the region of short wavelength (represented by the separate branches at the left of figs. 1 and 2) were made with solutions diluted to three times the original volume. It is apparent that only glucose and galactose, in this group of sugars, can be present in any considerable quantity in the carbohydrate in collagen. This result is in accord

4 In order to avoid repetitive use of the expression "solution containing the products of the reaction of sugar (or collagen) with phenol in a strongly acid medium," the phrase "sugar (or collagen) with phenol" will be substituted. 
with the report of Grassmann and Schleich [5] that the carbohydrate is composed of equal quantities of glucose and galactose. However, when an attempt was made to represent the absorbency of the collagen with orcinol as a multiple of the average for glucose and galactose, the result was not satisfactory. Table 1 gives the ratio of the absorbency for collagen to the average for glucose and galactose at a few wavelengths. The variation of this ratio is greater than the uncertainty of the measurement. If it is ascribed to the influence of the protein hydrolyzate on the formation of the colored substances, it

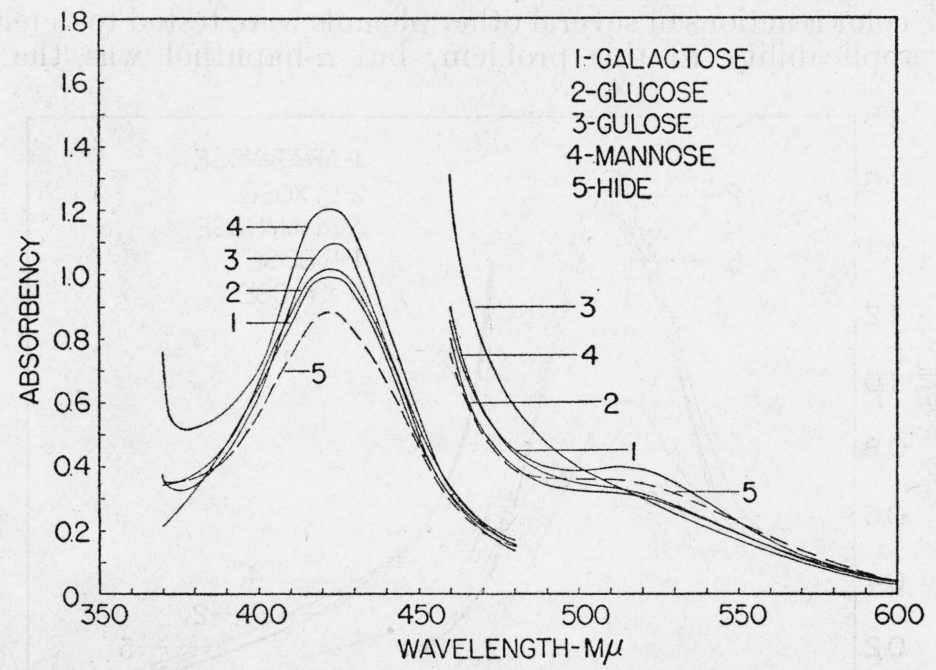

Figdre 2.-Spectral absorbency obtained with four aldohexoses"and with hide collagen, after heating with orcinol.

The solutions were diluted threefold before making the measurements at low wavelengths.

must be supposed that the effect is to decrease the amount of substance absorbing in the blue relative to the amount absorbing in the yellow.

TABLE 1. The ratio of the absorbency of collagen heated with orcinol to that of $a$ mixture of equal amounts of glucose and galactose heated with orcinol

\begin{tabular}{|c|c|}
\hline Wavelength & $\frac{2 \text { (Collagen) }}{\text { (Glucose) }+ \text { (Galactose) }}$ \\
\hline$m_{\mu}$ & \\
560 & 1.12 \\
520 & 1.01 \\
480 & 0.93 \\
440 & .88 \\
400 & .89 \\
\hline
\end{tabular}

The comparison between the collagens from the different sources was made directly by measuring the relative transmittancy of solutions heated with orcinol. All three comparisons were made by measurements in duplicate at 10 wavelengths. The differences in absorbency are shown in the second, third, and fourth columns of table 2. The fifth column gives the average of the three differences, which would be zero if the measurements were without error. Adjusted values of the differences were obtained by subtracting the 
figures in the fifth column from those in the preceding three. These adjusted values were used in conjunction with the absorbency of solutions of hide heated with orcinol to calculate the ratio of the concentration of carbohydrate in the solutions of tendon and bone to that in the solution of hide. The results are shown in the last two columns of table 2 .

TABLE 2.-A comparison of the carbohydrate contents of solutions of hide, tendon, and bone, using the absorbencies of solutions heated with orcinol

$[H, T$, and $B$ denote absorbencies obtained with hide, tendon, and bone, respectively. The concentration of collagen is slightly different in the three solutions.]

\begin{tabular}{|c|c|c|c|c|c|c|}
\hline $\begin{array}{l}\text { Wave- } \\
\text { length }\end{array}$ & $H-T$ & $T-B$ & $B-H$ & $\mathrm{Sum} / 3$ & $T / H$ & $B / H$ \\
\hline $\begin{array}{c}m \mu \\
550 \\
540 \\
530 \\
520 \\
510 \\
500 \\
490 \\
480 \\
470 \\
460\end{array}$ & $\begin{array}{r}0.033 \\
.049 \\
.062 \\
.066 \\
.060 \\
.057 \\
.050 \\
.054 \\
.064 \\
.074\end{array}$ & $\begin{array}{r}-0.035 \\
-.044 \\
-.059 \\
-.062 \\
-.066 \\
-.060 \\
-.056 \\
-.055 \\
-.060 \\
-.084\end{array}$ & $\begin{array}{r}0.004 \\
.005 \\
.006 \\
.006 \\
.005 \\
.002 \\
-.006 \\
-.008 \\
-.014 \\
-.032\end{array}$ & $\begin{array}{r}0.001 \\
.003 \\
.003 \\
.003 \\
.000 \\
.000 \\
-.004 \\
-.003 \\
-.003 \\
-.014\end{array}$ & $\begin{array}{l}0.86 \\
.83 \\
.81 \\
.82 \\
.83 \\
.84 \\
.85 \\
.85 \\
.87 \\
.88\end{array}$ & $\begin{array}{l}1.01 \\
1.01 \\
1.01 \\
1.01 \\
1.01 \\
1.01 \\
0.99 \\
.99 \\
.98 \\
.98\end{array}$ \\
\hline
\end{tabular}

The average values of $T / H$ and $B / H$ are 0.85 and 1.00 , respectively. The corresponding ratios of the weights of the samples are 0.90 and 1.02. Thus the apparent ratios of the concentrations of carbohydrate in the tendon and in the bone to that in the hide are 0.94 and 0.99 .

A similar comparison of tendon and hide was made, using the solutions prepared in sulfuric acid at $25^{\circ} \mathrm{C}$. The ratio obtained in this case was 0.97 , the fluctuations being somewhat less than those in table 2.

In view of the small variations in the ratios shown in table 2 , and of the small differences between the values obtained for the three preparations, it may be concluded that all three contain the same amount of the same kind of carbohydrate. The addition of this confirmatory evidence to that cited in the introduction makes possible the further conclusion that the white fibrous connective tissues of the hide, tendon, and bone of the steer are chemically identical.

The uncertainty about the qualitative composition of the carbohydrate mixture leaves some uncertainty about the quantity present. Although all the aldohexoses measured give approximately the same absorbency at $425 \mathrm{~m} \mu$, the measurements are subject to greater errors in this region of the spectrum, and it is also in this region that the protein hydrolyzate interferes most strongly with the measurement. The other colorimetric methods described in the experimental section were used to obtain information about the composition of the carbohydrate.

Figures 3,4 , and 5 show the curves for some known sugars, galacturonic acid, and hide collagen with sulfuric acid at $25^{\circ} \mathrm{C}$. The curve for collagen corresponds fairly well to that for a mixture of equal quantities of glucose and galactose, but the smaller absorption in the blue still persists.

Figures 6 and 7 show the curves for several known sugars and hide collagen with hydrochloric acid and ferric chloride. Here the results 
for collagen are intermediate between those for glucose and galactose, but the apparent proportion of glucose is larger than that indicated by the other experiments.

Figure 8 shows the transmittancies (referred to water) of glucose, galactose, and hide collagen with $\alpha$-naphthol. The shape of the

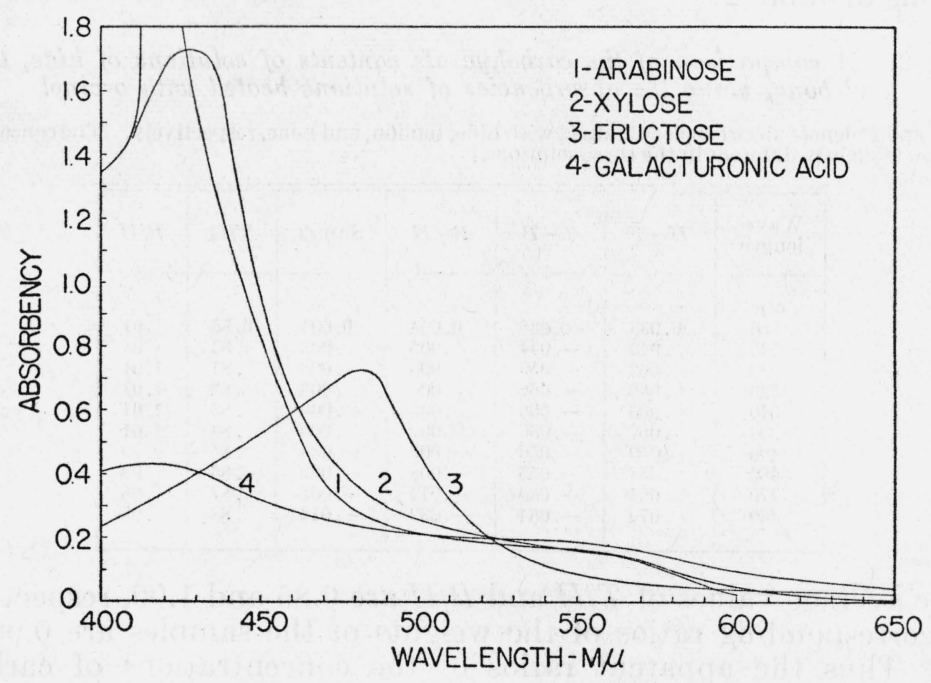

FIGURE 3.-Spectral absorbency obtained with three sugars and with galacturonic acid, after reaction with orcinol at $25^{\circ} \mathrm{C}$.

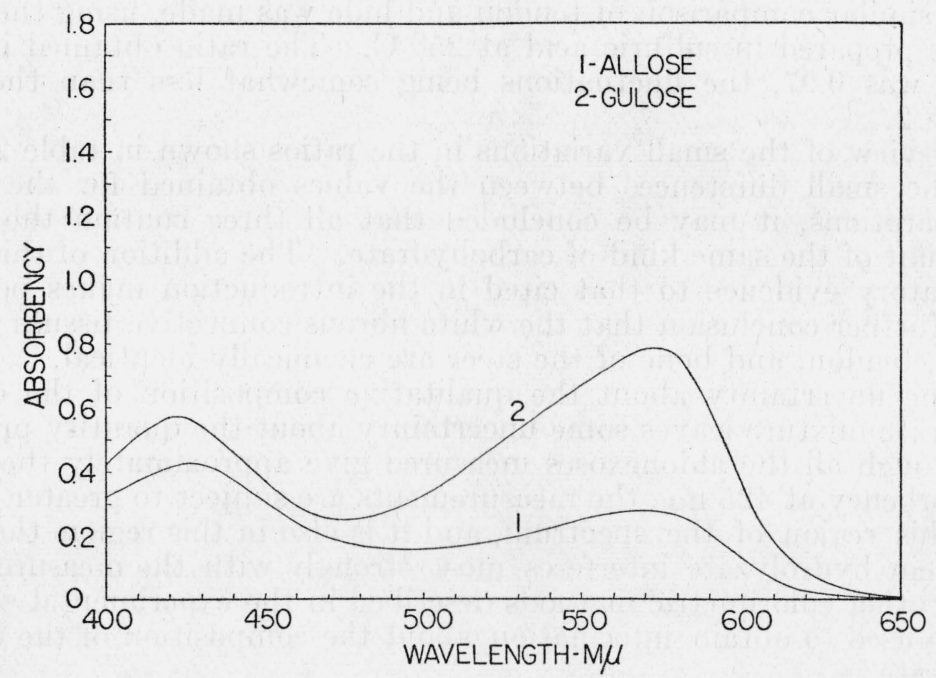

FIGURE 4.-Spectral absorbency obtained with two aldohexoses after reaction with orcinol at $25^{\circ} \mathrm{C}$.

curve for collagen corresponds to that for a mixture of glucose and galactose.

If the carbohydrate could be removed from the hydrolyzate, the interfering effect of the noncarbohydrate material could be determined by adding a known sugar and then carrying out the color reaction. 
It was suggested by H. S. Isbell, of the Polarimetry Section of the National Bureau of Standards, that a convenient way to destroy the carbohydrate without otherwise affecting the hydrolyzate would be to

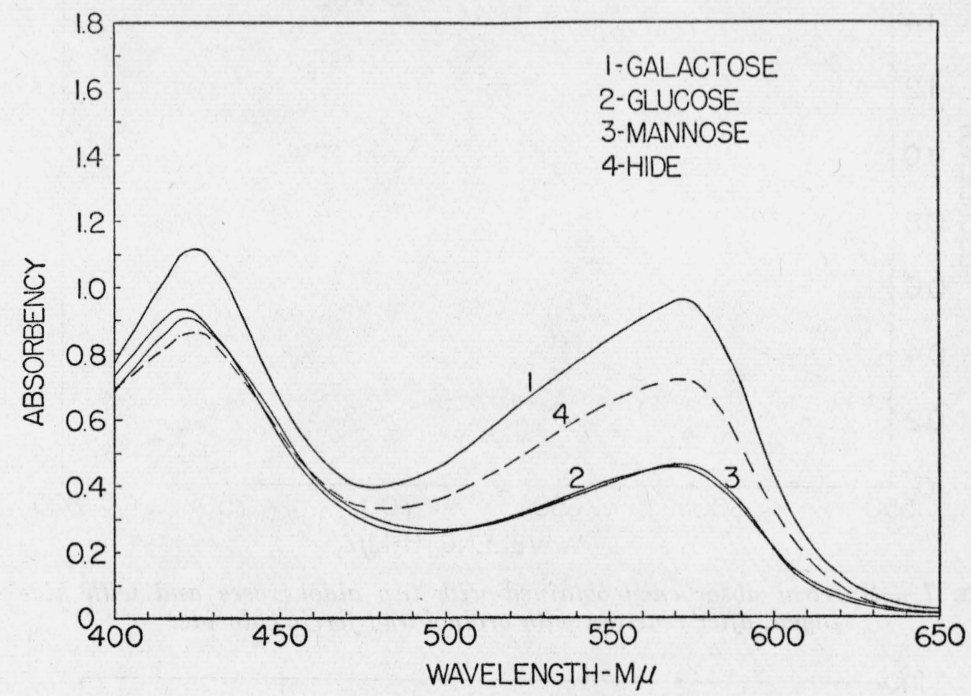

FIGURE 5.-Spectral absorbency obtained with three aldohexoses and with hide collagen, after reaction with orcinol at $25^{\circ} \mathrm{C}$.

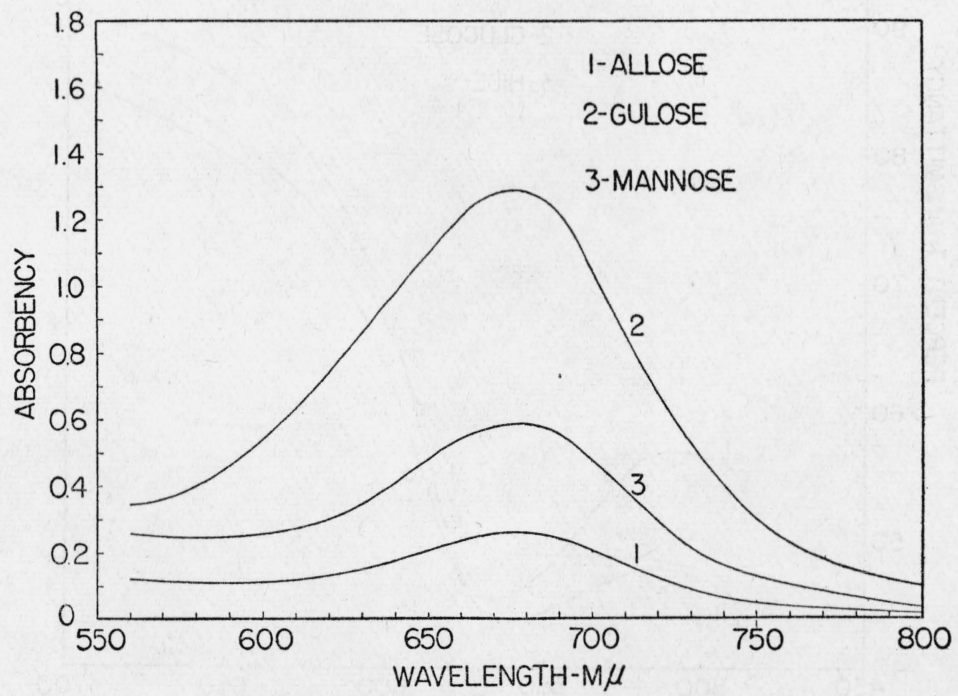

FIGURE 6.-Spectral absorbency obtained with three aldohexoses after reaction with orcinol and ferric chloride.

ferment the sugars with galactose-active yeast, provided, of course, that the carbohydrate is made up of $d$-glucose and $d$-galactose. Galactose-active yeast was prepared by a method similar to that of Kirby and Atkin [6]. Four successive cultures of yeast were grown in solu- 


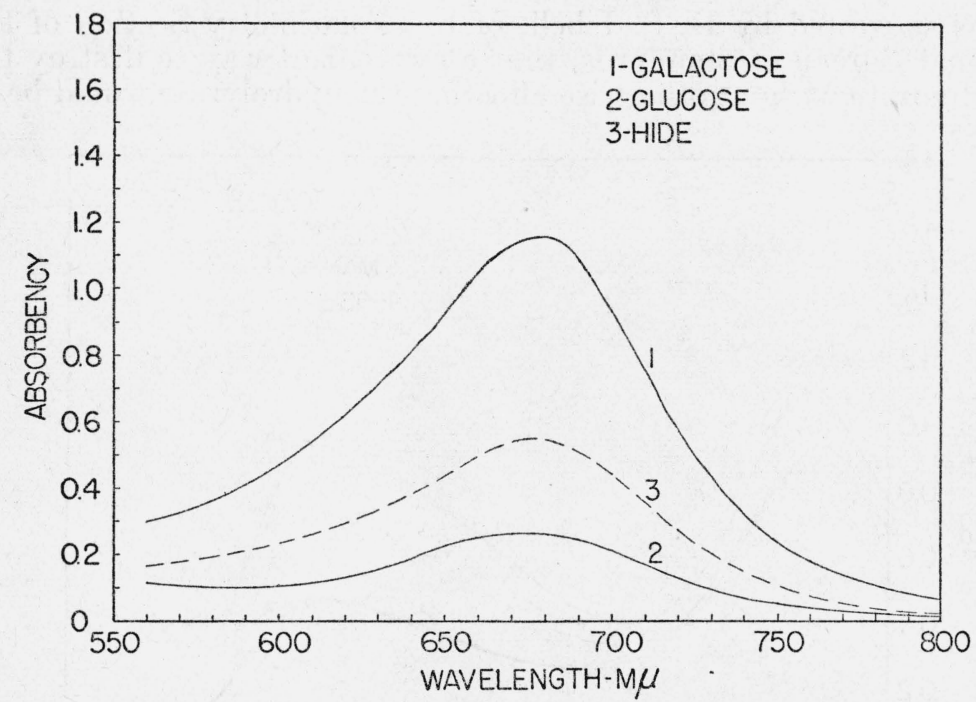

FIGURE 7.-Spectral absorbency obtained with two aldohexoses and with hide collagen, after reaction with orcinol and ferric chloride.

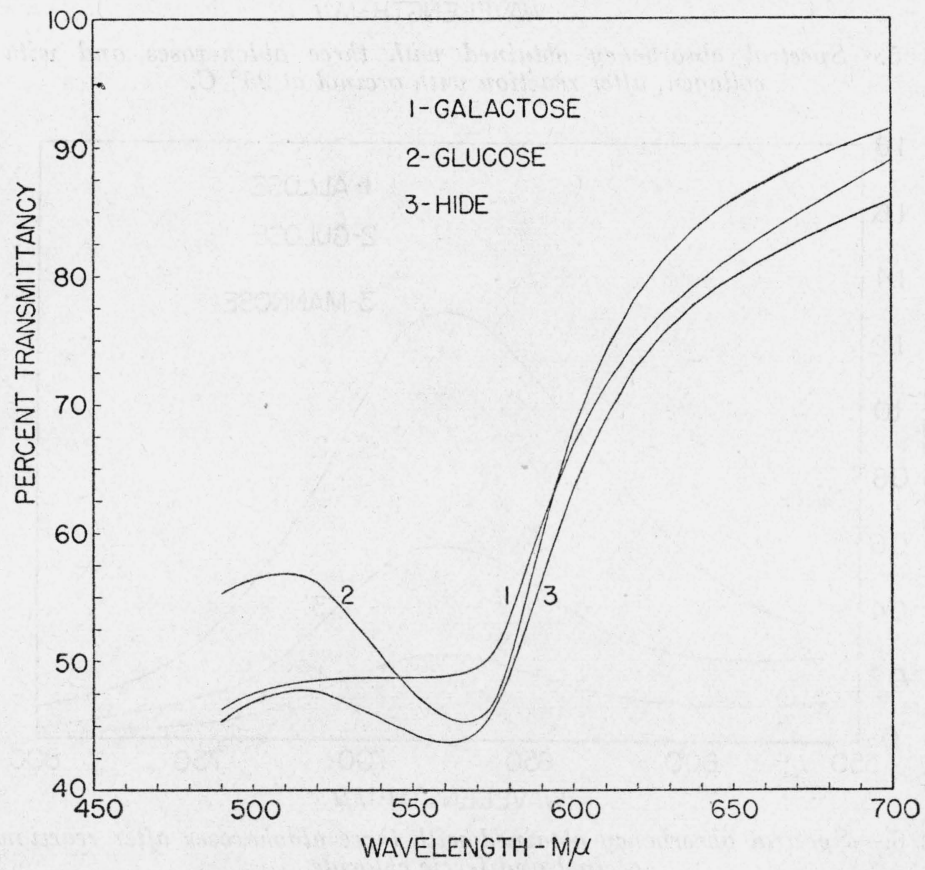

FIGURE 8.-Spectral transmittancy obtained with glucose, galactose, and hide collagen, after heating with $\alpha$-naphthol.

tions containing $d$-glucose and $d$-galactose. The final product was washed with nutrient solution by centrifugation.

An attempt was made to ferment the sugar in a neutral solution of hydrolyzate, but the sugar was not fermented. In order to make sure 
that the yeast was active in the solution in question, experiments were carried out in which $d$-galactose and yeast were added to hydrolyzed hide and bone collagens. In both cases the $d$-galactose was fermented and the sugar from the collagen remained. Table 3 gives the transmittancies of hide collagen and orcinol at $25^{\circ} \mathrm{C}$ with and without added galactose, after incubation with galactose-active yeast at $25^{\circ} \mathrm{C}$ for 4 days. The amount of galactose added was approximately equivalent to the carbohydrate in the collagen.

TABLE 3.-The percentage transmittancies of collagen hydrolyzate with orcinol with and without added d-galactose, after incubation with galactose-active yeast

\begin{tabular}{|c|c|c|}
\hline Wavelength & Hide collagen $+d$-galactose & Hide collagen \\
\cline { 2 - 3 } & & \\
\hline$m \mu$ & 95.4 & 95.1 \\
650 & 54.1 & 56.6 \\
500 & 25.4 & 28.0 \\
550 & 29.4 & 31.5 \\
500 & 44.0 & 44.4 \\
480 & 47.5 & 26.2 \\
450 & 27.8 & 9.6 \\
420 & 10.8 & \\
\hline
\end{tabular}

As the carbohydrate in the hydrolyzed collagen was not fermented, its identity was not established. If the sugar could be separated from most of the hydrolyzate, it might be expected that interference with the color reactions would be decreased. Rimington [7] has given a method for concentrating the carbohydrate complex in proteins, and Schneider [8] has shown that the method is applicable to collagen. Accordingly, the first precipitation of Rimington's method was carried out, using as the starting material $300 \mathrm{~g}$ of American standard hide powder. Measurements were made on the product with orcinol in sulfuric acid at $25^{\circ} \mathrm{C}$, and with orcinol and ferric chloride in hydrochloric acid. The results are shown in figure 9. The curves in this figure represent the results calculated for a mixture of equal parts of glucose and galactose. The agreement between the two sets of results is close, and this agreement is significant because the ratio of the absorbency for galactose to that for glucose covers such a wide range. The ratio is 1.2 at $420 \mathrm{~m} \mu$ and 2.1 at $570 \mathrm{~m} \mu$ with sulfuric acid, and 4.4 at $680 \mathrm{~m} \mu$ with ferric chloride and hydrochloric acid. It may be concluded, then, that these results confirm the report of Grassmann and Schleich [5], although a more certain determination of the composition of the carbohydrate might be desired. The color reactions and the resistance to fermentation indicate either that the carbohydrate is bound by a very difficultly hydrolyzable linkage or that it is composed of $l$-glucose and $l$-galactose. Bell and Baldwin [9] recently reported finding $l$-galactose in the tissues of a common edible snail.

The amount of carbohydrate in the collagen may be calculated on the assumption that it is composed of equal parts of glucose and galactose. The experimental results are better explained on the whole by supposing that the ratio of galactose to glucose is about 3 to 2 , but the greater a priori likelihood of the ratio 1 to 1 makes its use seem more reasonable. The measurements in the neighborhood of $570 \mathrm{~m} \mu$ with sulfuric acid at $25^{\circ} \mathrm{C}$ were used for the calculation, because these measurements are most likely to be free from the effects 
of interference. The result for the hide collagen is 0.029 millimole of hexose per gram of dry collagen, which corresponds to 0.52 percent of monosaccharide, or 0.47 percent of carbohydrate residue, assuming that one molecule of water is eliminated in attaching each hexose residue. It may be pointed out that 0.029 millimole per gram gives a unit weight of about 34,000 , which is approximately the unit weight found for the globular protems. The coincidence is interesting even if not significant.

The form of the curves in figures 4 and 5 suggests the possibility that two colored substances are formed at different rates in the reaction of

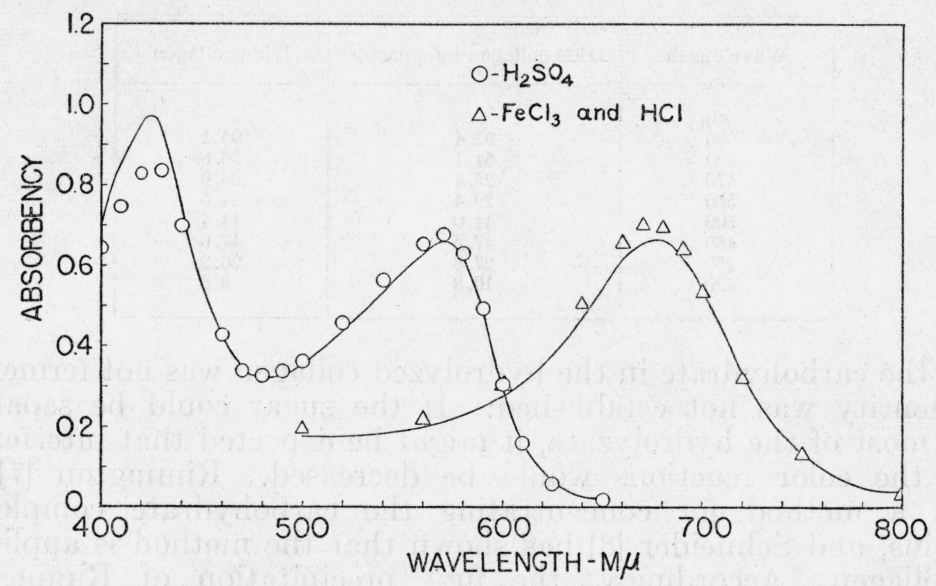

FIGURE 9.-Spectral absorbency obtained with the carbohydrate concentrate from hide powder, after reaction with orcinol, and with orcinol and ferric chloride at $25^{\circ} \mathrm{C}$.

The ordinates of the smooth curves were calculated to represent a mixture of equal quantities of glucose and galactose.

aldohexoses with orcinol in sulfuric acid at $25^{\circ} \mathrm{C}$, giving different ratios of the absorbency in the yellow band to that in the blue band. An attempt was made to represent the absorbencies of all the sugars as the sum of two component absorbencies, which are supposed to arise from the presence of the same two substances in different amounts. At wavelengths greater than $520 \mathrm{~m} \mu$, the ratios of the absorbencies of the various sugars are nearly constant, so that these ratios may be taken as the ratios of the amounts of the substance absorbing in the yellow. It was not possible, however, to represent the absorbencies in the green and blue as arising from only two substances. ${ }^{5}$ Thus at least three absorption bands are involved in the observed color.

If it is assumed that no substance with more than one absorption band in the visible region is formed in the color reaction, a lower bound for the efficiency of the absorption process may be calculated from the total concentration of sugar and the integral of the absorbency with respect to the frequency. This lower bound in turn permits an estimate to be made of a lower bound for the amount of sugar that is converted to colored substances, as it is impossible for the efficiency to be greater than unity, and it is reasonable to assume that it is not

\footnotetext{
$\checkmark$ That is, if the absorbency for the $i$ th sugar is represented as $F_{i}(\lambda)$, and $a_{i}$ and $b_{i}$ are constants characteristic of the ith sugar, no two functions $f_{a}(\lambda)$ and $f_{b}(\lambda)$ exist, such that the equations $F_{i}(\lambda)=a_{i} f_{a}(\lambda)+b_{i} f_{b}(\lambda)$ are satisfied for all values of $i$.
} 
greater than 0.6 (for example, the efficiency for methylene blue at $\mathrm{pH} 3.4$ in water is 0.45 [10]). The apparent efficiencies were calculated for galactose with sulfuric acid at $25^{\circ} \mathrm{C}$, and for gulose with ferric chloride and hydrochloric acid. The results were 0.4 and 0.2 , respectively. Thus the efficiencies of the two absorption processes are at least 0.4 and 0.2 , and it may be supposed that at least 0.7 and 0.3 of the respective sugars are converted to colored substances.

\section{SUMMARY}

Three colorimetric methods are used to investigate the carbohydrate in collagen from hide, tendon, and bone. The results indicate that the three collagen preparations all contain the same amount of the same kind of carbohydrate. With this evidence in confirmation of results of other comparisons, it may be concluded that the white fibrous connective tissues of the hide, tendon, and bone of the steer are chemically identical. By the use of a fermentation technique, it is demonstrated that the collagen hydrolyzate contains no considerable amount of either free $d$-glucose or free $d$-galactose. The color reactions of a concentrate of the carbohydrate complex are closely similar to those of a mixture of equal quantities of glucose and galactose, which indicates that the carbohydrate either is bound by a difficultly hydrolyzable linkage or is composed of a mixture of $l$-glucose and $l$-galactose. A lower bound for the efficiency of the absorption of light by the colored substances formed from certain sugars was calculated. The result shows that a large fraction of the sugar was converted to the colored substances by the reaction.

\section{REFERENCES}

[1] J. Research NBS 21, 117 (1938) RP1119; J. Am. Leather Chem. Assn. 33, 621 (1938).

[2] J. Research NBS 23, 271 (1939) RP1230; J. Am. Leather Chem. Assn. 34, 641 (1939).

[3] J. Am. Leather Chem. Assn. 31, 93 (1936).

[4] Compt. rend. trav. lab. Carlsberg, Sér. chim. 19, No. 12 (1933).

[5] Biochem. Z. 27y, 320 (1935).

[6] J. Biol. Chem. 116, 511 (1936).

[7] Biochem. J. 25, 1062 (1931).

[8] Collegium 1940, 97.

[9] Nature 146, 559 (1940).

[10] J. Am. Chem. Soc. 63, 69 (1941).

Washington, October 21, 1941. 